Stage 1 Protocol Accepted in Principle at Evolutionary Human Sciences.

Is vocal attractiveness a cue of young adult women's susceptibility to infectious illnesses?

Kieran J. O'Shea ${ }^{1}$, Amanda C. Hahn ${ }^{2}$, Rebecca J. Lai ${ }^{1}$, Iris J. Holzleitner ${ }^{1}$, Vanessa Fasolt ${ }^{1}$, Anthony J. Lee ${ }^{3}$, Christoph Schild ${ }^{4}$, Rachel Cassar ${ }^{1}$, David R. Feinberg ${ }^{5}$, Lisa M. DeBruine ${ }^{1} \&$ Benedict C. Jones ${ }^{1}$

${ }^{1}$ Institute of Neuroscience and Psychology, University of Glasgow, Glasgow Scotland, UK.

${ }^{2}$ Department of Psychology, Humboldt State University, Arcata, California, USA.

${ }^{3}$ Division of Psychology, University of Stirling, Stirling, Scotland, UK.

${ }^{4}$ Department of Psychology, University of Copenhagen, Copenhagen, Denmark.

${ }^{5}$ Department of Psychology, Neuroscience and Behaviour, McMaster

University, Hamilton, Canada.

Corresponding author: Kieran J. O'Shea (Kieran.O'Shea@glasgow.ac.uk), Institute of Neuroscience \& Psychology, University of Glasgow, Scotland, UK.

Acknowledgements: This research was supported by European Research Council grants awarded to BCJ (OCMATE) and LMD (KINSHIP). 


\title{
Is vocal attractiveness a cue of young adult women's susceptibility to infectious illnesses?
}

\begin{abstract}
Although many researchers have assumed that vocal attractiveness functions as a health cue, direct tests of this assumption have been rare. Consequently, we will test possible correlations between women's vocal attractiveness and susceptibility to infectious illnesses. We will test correlations between women's vocal attractiveness and both a range of self-report measures of susceptibility to infectious illnesses and a commonly used salivary biomarker of immune response (secretary immunoglobulin $A$, measured from saliva).
\end{abstract}

\section{Introduction}

Previous research on vocal attractiveness (the perceived attractiveness of voices when isolated from other cues, such as visual or olfactory cues) has suggested that vocal attractiveness plays a role in mate choice in humans (e.g., Apicella et al., 2007; Hill et al., 2013; Leongómez et al., 2014). For example, individuals possessing vocal characteristics that are correlated with attractiveness report greater reproductive potential (as indexed by reported number of sexual partners, Kordsmeyer et al., 2018, Hill et al., 2013) and, in hunter-gatherers, have greater reproductive fitness (Apicella et al., 2007). People also alter their vocal attractiveness in mating contexts, such as when interacting with an attractive potential mate (Leongómez et al., 2014; Pisanksi et al., 2018).

The function of vocal attractiveness judgments is unclear. One influential theory is that vocal attractiveness functions as a cue of susceptibility to infectious illnesses (Arnocky et al., 2018; Hughes et al., 2002). Under this theory, vocal attractiveness is thought to be a cue of low susceptibility to infectious illnesses and, by extension, heritable immunocompetence (Arnocky et al., 2018; Hughes et al., 2002). Thus, preferences for potential mates with attractive voices may be adaptive as it may increase offspring health and reproductive fitness (Arnocky et al., 2018; Hughes et al., 2002).

Although a popular assumption, there have been very few direct tests of the possible relationships between vocal attractiveness and susceptibility to infectious illnesses in humans (Arnocky et al., 2018). Arnocky et al. (2018) reported that an attractive characteristic in men's voices (low voice pitch) was positively correlated with their salivary secretary immunoglobulin A (SIgA) and negatively correlated with a self-report measure of their susceptibility to infectious illnesses. SIgA is the main immunoglobulin in mucous secretions from the salivary glands and is widely used as a marker for immune function (Higham et al., 2010; Van Anders, 2010) because it acts as a defense against microbial invasion (Bosch et al., 2011; Mantis et al., 2011).

While Arnocky et al. (2018) interpreted their results for vocal attractiveness as evidence that vocal attractiveness was a health cue, the correlations reported were two out of 66 correlations with no correction for multiple comparisons. Indeed, the correlations between attractiveness and health would not have 
been significant if alpha was corrected for these multiple comparisons. Thus, we suggest these results be treated cautiously.

Other work has reported that women with greater body asymmetry have less attractive voices (Hughes et al., 2002). Hughes et al. (2002) interpreted this finding as evidence that vocal attractiveness is a cue of women's susceptibility to infectious illnesses, based on the assumption that, because degree of symmetry reflects developmental stability, it will be an index of susceptibility to infectious illnesses (Thornhill \& Gangestad, 1999). However, empirical research on this issue in humans suggests that there is little evidence that asymmetry is reliably related to susceptibility to infectious illnesses in humans (see Van Dongen \& Gangestad, 2011 for meta-analytic evidence that symmetry is not reliably associated with health outcomes in humans).

In light of the above, we will test for possible correlations between measures of susceptibility to infectious illnesses and women's vocal attractiveness. We will test correlations between vocal attractiveness and both a range of selfreport measures of susceptibility to infectious illnesses and SIgA. We focused on women's vocal attractiveness because Arnocky et al. (2018) focused on men's vocal attractiveness and because we had a large sample of women's voices collected in a previous study (see Methods).

\section{Predictions}

Prediction 1. Women with more attractive voices will report better health as assessed by Stevenson et al's (2009) infection frequency and recency subscales, Wilson et al's (2005) Upper Respiratory Illness Scale, or Duncan et al's (2009) Perceived Vulnerability to Disease scale.

Prediction 2. Women with more attractive voices will have higher levels of Secretory Immunoglobulin A (SlgA).

\section{Methods}

\section{Ethics}

All aspects of this study have been approved by University of Aberdeen, Psychology Ethics Committee.

\section{Sample and justification of sample size}

Data were collected from 344 young adult women (mean age $=21.1$ years, SD $=3.19$ years). Hughes et al. (2002) investigated putative health correlates of vocal attractiveness in a sample of 46 women and 50 men. Arnocky et al. (2018) investigated putative health correlates of vocal attractiveness in a sample of 108 men. Our sample is then considerably larger than previous work on the topic. Data were collected as part of a larger study of putative hormonal effects on women's behavior (Jones et al., 2018a). Voice recordings have already been collected, but have not yet been analyzed or rated.

\section{Health questionnaires}

Women were asked to complete Stevenson et al's (2009) infection frequency and recency questionnaire, which consists of two subscales that assess the frequency with which the participant has suffered from different infectious 
illnesses (e.g. colds, ear infections) in the previous year (the infection frequency subscale) and how long ago the most recent occurrence of each infectious illness was (the infection recency subscale). Fifteen women chose not to complete the infection frequency subscale and 3 women chose not to complete the infection recency subscale. Women were also asked to complete a version of Wilson et al's (2005) Upper Respiratory IIIness Scale, which assesses the frequency with which participants had suffered from ten symptoms of upper respiratory illness (e.g. sore throat, coughing) in the previous week, and Duncan et al's (2009) Perceived Vulnerability to Disease Scale. This scale assesses perceptions of one's own susceptibility to infectious illnesses and discomfort of situations that increase likelihood of pathogen exposure. Higher scores on each of these scales indicate poorer health. Six women chose not to complete the Upper Respiratory IIIness Scale and 7 women chose not to complete the Perceived Vulnerability to Disease Scale. These health questionnaires have previously been used to assess the relationship between women's facial attractiveness and susceptibility to infectious illnesses in Cai et al. (2018).

\section{Secretory Immunoglobulin A (SIgA)}

Each woman provided a saliva sample via passive drool (Papacosta \& Nassis, 2011). Participants were instructed to avoid consuming alcohol and coffee in the 12 hours prior to participation and avoid eating, smoking, drinking, chewing gum, or brushing their teeth in the 60 minutes prior to participation. Saliva samples were frozen immediately and stored at $-32^{\circ} \mathrm{C}$ until being shipped on dry ice to the Salimetrics Lab (Suffolk UK) for analysis where they were assayed using their Salivary Secretory IgA Enzyme Immunoassay Kit 1-1602. Only 153 women's saliva samples were analyzed for SIgA. Following recommendations by Salimetrics Lab, we analyzed SIgA corrected for flow rate (see also Arnocky et al., 2018).

\section{Voice recordings}

A mono digital voice recording was taken from each woman using an AudioTechnica AT-4041 cardioid condenser microphone at a sampling rate of 44.1 $\mathrm{kHz}$ at 16-bit amplitude quantization. Each participant was instructed to say "A-E-I-O-U" in their normal speaking voice.

\section{Attractiveness ratings}

The sound pressure level of all voices will be amplitude normalized to $70 \mathrm{~dB}$ using the root mean squared method (following Armstrong et al., 2019). Voice recordings will then be presented via headphones and all raters will rate each voice individually on attractiveness using a 1 (very unattractive) to 7 (very attractive) scale. Trial order will be fully randomized, and participants will not be able to rate a voice until it has been played in full length. Participants will be able to listen to a voice as many times as they like before rating it and will be instructed to take regular breaks during the rating task to avoid rater fatigue. Participants will start each trial by clicking 'play'. Figure 1 shows a screen capture of the rating interface. Fifteen heterosexual men and 15 heterosexual women (all between 16 and 35 years of age) will rate the voices. Simulations have shown that 15 raters is typically sufficient to obtain high inter-rater agreement for attractiveness ratings (https://osf.io/x7fus/). 


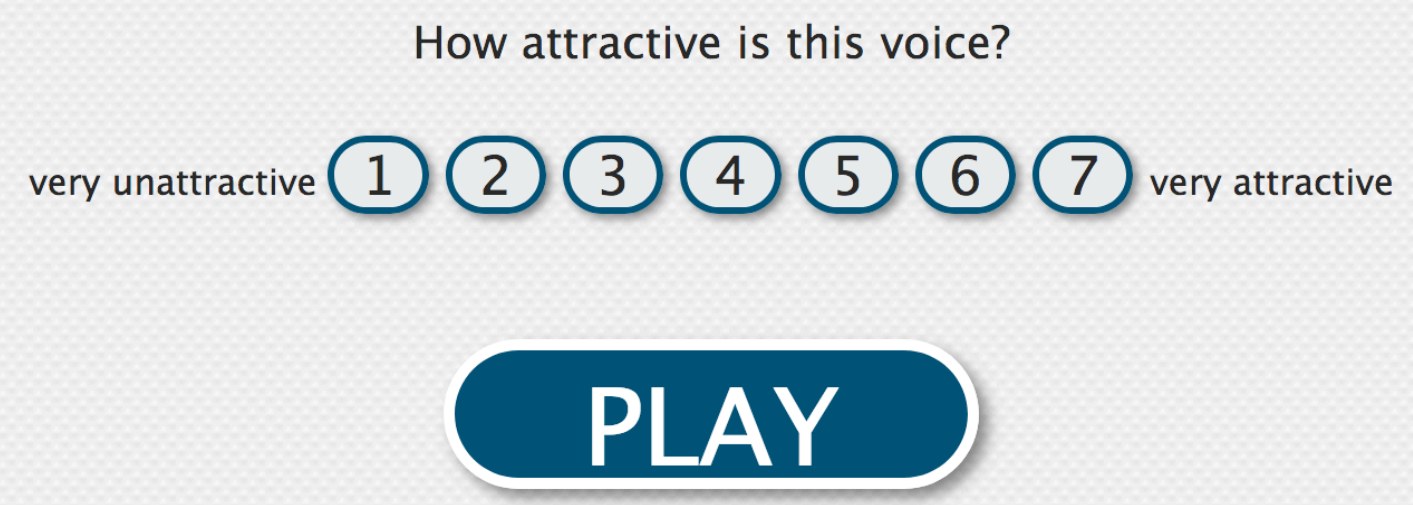

Figure 1. Interface for collecting vocal attractiveness ratings.

\section{Data quality checks and exclusions}

Raters who give the same rating to $75 \%$ or more of the voices will be excluded from the dataset prior to any data analyses. There will be no other exclusions. If inter-rater agreement (estimated from Cronbach's alpha) for attractiveness ratings is $<.8$, more raters will be added until Cronbach's alpha for attractiveness ratings is $>.8$. Ratings by men and women will be combined (i.e., averaged) only if the correlation between men's and women's attractiveness ratings is greater than .80. Otherwise, men's and women's ratings will be analyzed separately, with more raters added until the relevant Cronbach's alpha for attractiveness ratings is $>.8$.

\section{Analysis plan}

To minimize the effects of extreme values and outliers, we will test the relationships between vocal attractiveness and each of the various health measures by calculating Spearman's rho. Mean attractiveness ratings will serve as the outcome measure in each analysis. Analysis code (with simulated data) is publicly available at https://osf.io/yj7 rp/ and in our supplemental materials.

Prediction 1. Women with more attractive voices will report better health as assessed by Stevenson et al's (2009) infection frequency and recency subscales, Wilson et al's (2005) Upper Respiratory Illness Scale, or Duncan et al's (2009) Perceived Vulnerability to Disease scale.

This prediction will be supported if mean attractiveness ratings and scores on at least one of these four questionnaires are negatively and significantly correlated. The critical alpha will be set at .0125 to correct for multiple comparisons. We have $90 \%$ power to detect absolute $r$ values of $.206, .203$, .203, and .204 for Stevenson et al's (2009) infection frequency and recency subscales, the Upper Respiratory Illness Scale, and the Perceived Vulnerability to Disease scale, respectively.

Prediction 2. Women with more attractive voices will have higher levels of Secretory Immunoglobulin A (SIgA). 
This prediction will be supported if mean attractiveness ratings and SIgA are positively and significantly correlated. The critical alpha will be set at .05 . We have $90 \%$ power to detect an absolute $r$ value of .258 .

For both predictions, if the correlations are small (i.e., $r<|.30|$ ), we will note in our Discussion that this raises questions about their biological significance.

\section{Robustness checks}

In addition to the analyses described above, we will repeat each analysis using non-parametric partial correlations controlling for women's age. Code for these analyses is publicly available at https://osf.io/yj7rp/ and in our supplemental materials.

\section{Additional exploratory analyses}

We will also report results of exploratory analyses testing for correlations among the various health measures.

\section{References}

Apicella, C. L., Feinberg, D. R., \& Marlowe, F. W. (2007). Voice pitch predicts reproductive success in male hunter-gatherers. Biology Letters, 3, 682684.

Armstrong, M. M., Lee, A. J., \& Feinberg, D. R. (2019). House of cards: false perception of body size mediates the relationship between voice pitch and perceptions of dominance. Animal Behaviour, 147, 43-51.

Arnocky, S., Hodges-Simeon, C., Ouellette, D. \& Albert, G. (2018). Do men with more masculine voices have better immunocompetence? Evolution and Human Behavior, 39, 602-610.

Bosch, J. A., Ring, C. de Geus, E. J., Veerman, E. C. \& Amerongen, A. V. N. (2002). Stress and secretory immunity. International Review of Neurobiology, 52, 213-253.

Cai, Z., Hahn, A. C., Zhang, W., Holzleitner, I. J., Lee, A. J., DeBruine, L. M., \& Jones, B. C. (2018). No evidence that facial attractiveness, femininity, averageness, or coloration are cues to susceptibility to infectious illnesses in a university sample of young adult women. Evolution and Human Behavior.

Duncan, L.A., Schaller, M., Park, J.H. (2009). Perceived vulnerability to disease: Development and validation of a 15-item self-report instrument. Personality and Individual differences, 47, 541-546.

Higham, J.P., Vitale, A.B., Rivera, A.M., Ayala, J.E., \& Maestripieri, D. (2010). Measuring salivary analytes from free-ranging monkeys. Physiology \& Behavior, 101 601-607.

Hill, A. K., Hunt, J., Welling, L. L. M., Cárdenas, R. A., Rotella, M. A., Wheatley, J. R., ... Puts, D. A. (2013). Quantifying the strength and form of sexual selection on men's traits. Evolution and Human Behavior, 34, 334-341. 
Hughes, S. M., Harrison, M. A., \& Gallup Jr, G. G. (2002). The sound of symmetry: Voice as a marker of developmental instability. Evolution and Human Behavior, 23, 173-180.

Jones, B. C., Hahn, A. C., Fisher, C. I., Wang, H., Kandrik, M., Han, C., ... DeBruine, L. M. (2018a). No compelling evidence that preferences for facial masculinity track changes in women's hormonal status. Psychological Science, 29, 996-1005.

Jones, B. C., Hahn, A. C., Fisher, C. I., Wang, H., Kandrik, M., Lao, J., ... \& DeBruine, L. M. (2018b). No compelling evidence that more physically attractive women have higher estradiol or progesterone. Psychoneuroendocrinology, 98, 1-5.

Kordsmeyer, T. L., Hunt, J., Puts, D. A., Ostner, J., \& Penke, L. (2018). The relative importance of intra-and intersexual selection on human male sexually dimorphic traits. Evolution and Human Behavior, 39(4), 424436.

Leongómez, J. D., Binter, J., Kubicová, L., Stolařová, P., Klapilová, K., Havlíček, J., \& Roberts, S. C. (2014). Vocal modulation during courtship increases proceptivity even in naive listeners. Evolution and Human Behavior, 35, 489-496.

Mantis, N.J., Rol, N., Corthésy, B. (2011). Secretory IgA's complex roles in immunity and mucosal homeostasis in the gut. Mucosal immunology, 4, 603.

Papacosta, E., \& Nassis, G.P. (2011). Saliva as a tool for monitoring steroid peptide and immune markers in sport and exercise science. Journal of Science \& Medicine in Sport, 14, 424-434.

Pisanski, K., Oleszkiewicz, A., Plachetka, J., Gmiterek, M., \& Reby, D. (2018). Voice pitch modulation in human mate choice. Proceedings of the Royal Society B, 285(1893), 20181634.

Simonsohn, U. (2015). Small telescopes: Detectability and the evaluation of replication results. Psychological Science, 26, 559-569.

Stevenson, R.J., Case, T.I., \& Oaten, M.J. (2009). Frequency and recency of infection and their relationship with disgust and contamination sensitivity. Evolution and Human Behavior, 30, 363-368.

Thornhill, R., \& Gangestad, S.W. (1999). Facial attractiveness. Trends in Cognitive Science, 3, 452-460.

Van Anders, S.M. (2010). Gonadal steroids and salivary IgA in healthy young women and men. American Journal of Human Biology, 22 348-352.

Wilson, W.C., Rosenthal, B.S., Austin, S. (2005). Exposure to community violence and upper respiratory illness in older adolescents. Journal of Adolescent Health, 36, 313-319. 